\title{
Fluid Simulation by the Smoothed Particle Hydrodynamics Method: A Survey
}

\author{
T. Weaver and Z. Xiao \\ Centre for Digital Entertainment, National Centre for Computer Animation, Bournemouth University, United Kingdom \\ $\{$ tweaver, zxiao\}@bournemouth.ac.uk
}

\begin{abstract}
Keywords: $\quad$ SPH, Computer Graphics, GPU, CUDA, CFD, Physically-based simulation, Fluid simulation.
Abstract: $\quad$ This paper presents a survey of Smoothed Particle Hydrodynamics (SPH) and its use in computational fluid dynamics. As a truly mesh-free particle method based upon the Lagrangian formulation, SPH has been applied to a variety of different areas in science, computer graphics and engineering. It has been established as a popular technique for fluid based simulations, and has been extended to successfully simulate various phenomena such as multi-phase flows, rigid and elastic solids, and fluid features such as air bubbles and foam. Various aspects of the method will be discussed: Similarities, advantages and disadvantages in comparison to Eulerian methods; Fundamentals of the SPH method; The use of SPH in fluid simulation; The current trends in SPH. The paper ends with some concluding remarks about the use of SPH in fluid simulations, including some of the more apparent problems, and a discussion on prospects for future work.
\end{abstract}

\section{INTRODUCTION}

Fluid simulation is utilised in many applications including video games, special effects in both film and television, medical and military simulations, and virtual reality. Some applications use a technique known as offline rendering, where the scene or effect is rendered by a large array of computers prior to its use in the application. This can take a varying amount of time depending on the complexity of the simulation, and how many particles are used. The simulation of fluids in real-time is a challenging area, with early examples using SPH reproducing relatively simple scenes such as calm bodies of water, gas interaction, and static smoke or fog. More recent implementations consider more of the properties that influence fluid behaviour such as splashing, bubbles, correct pressure representation, mixing of different fluid viscosities, and boiling and evaporation. As the available computing power has increased, achieving reliable frame rates in real-time simulations has become possible. However, because the complexity of the simulations has also increased there is still many areas that could benefit from research and refinement.

The development of parallel computing, through Application Programming Interfaces (APIs) such as CUDA and OpenCL, has been widely accepted by researchers for use in fluid simulation. In general terms, a simulation is made up of $n$ particles, and for each particle a set of equations must be solved each frame before the simulation can be updated and rendered. Solving the equations on the CPU means that these calculations are solved linearly, one after another, which for large values of $n$ would heavily increase the time taken to process each update. Utilising parallel computing means that for any number of $n$ particles, each particle equation could be solved in parallel, reducing the time taken to perform all calculations. Furthermore, some of the more complex processes used in fluid simulation such as Nearest Neighbour searching, and surface reconstruction/extraction can be executed entirely on the GPU, discussed further in Section 4.2.

Smoothed Particle Hydrodynamics is one of the most popular methods in physically-based fluid simulation. As the subject of this paper, SPH was chosen for a number of reasons: realistic simulations in real-time are achievable; a number of commercial 3D graphical applications for simulating fluid already use $\mathrm{SPH}$; and it is not only limited to fluid based applications, some recent research using SPH include the calculation of protein-ligand binding rates in BioPhysics (Pan et al., 2015), modelling the sound of a rigid body falling on water in Acoustics (Zhang et al., 2015), and for the estimation of sea wave impact on coastal structures in Coastal Engineering (Altomare et al., 2015). 


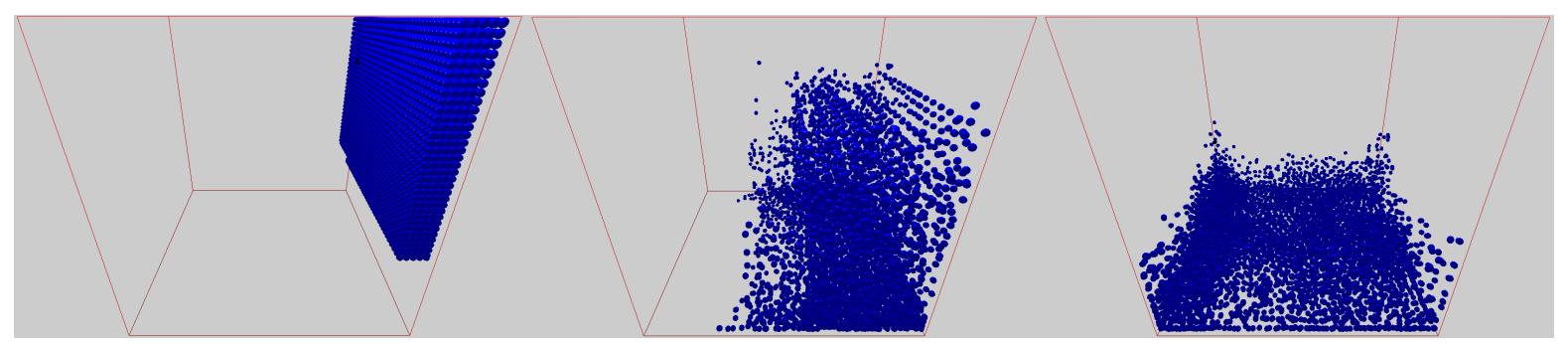

Figure 1: SPH simulation of a dam-break using 5000 particles executed entirely on the CPU averaging $40 \mathrm{fps}$.

\section{Smoothed Particle Hydrodynamics}

Smoothed Particle Hydrodynamics, herein referred to as SPH, was outlined in a series of papers by (Lucy, 1977) and (Gingold and Monaghan, 1977) to simulate nonaxisymmetric phenomena in astrophysics. Using $\mathrm{SPH}$, the authors were able to calculate spatial derivatives using analytical differentiation of interpolation formulae. This offered a vast improvement over other methods such as Particle in Cell, which utilises a grid structure in order to calculate the spatial derivatives. Additionally, specific particle attributes such as momentum, or pressure gradients, are found by sets of differential equations with the latter being calculated as the force between particle pairs.

Due to the extensibility and adaptability of SPH it has been widely adopted to realistically simulate a wide array of different fluid simulations and fluid phenomena such as the experiment illustrated in Figure 1. The next section discusses some key factors to consider when implementing SPH, including kernel functions, smoothing radius, boundary conditions, nearest neighbour searching, and time step evaluation.

\subsection{SPH Fundamentals}

As previously stated SPH is an interpolation method, which uses a set of disordered points (the particles) to express a function in terms of its values. The integral interpolant of any function $A(\mathbf{r})$ is defined by

$$
A_{I}(\mathbf{r})=\int_{\Omega} A\left(\mathbf{r}^{\prime}\right) W\left(\mathbf{r}-\mathbf{r}^{\prime}, h\right) \mathbf{d} \mathbf{r}^{\prime}
$$

where the integration is over the entire space $\Omega, \mathbf{r}$ is any point in the space, and $W$ is a smoothing kernel with $h$ width.

The integral interpolant can also be approximated by a summation interpolant, given by

$$
A_{S}(\mathbf{r})=\sum_{b} m_{b} \frac{A_{b}}{\rho_{b}} W\left(\mathbf{r}-\mathbf{r}_{b}, h\right)
$$

where $b$ denotes a particle, and the summation is over all particles. Particle $b$ has the following attributes: mass $m_{b}$, position $\mathbf{r}_{b}$, density $\rho_{b}$, velocity $\mathbf{v}_{b}$, and the value of any quantity $A$ at $\mathbf{r}_{b}$ is denoted by $A_{b}$.

It is important to understand that this means a differentiable interpolant of a function can be constructed from its values at the particles (interpolation points), by using a kernel that is differentiable (Monaghan, 1992). Thus any derivatives of this interpolant can be obtained using standard differentiation, which infers there is no need for grids or finite difference methods.

Some "golden" rules to be considered in the SPH method were presented in the early stages of research (Monaghan, 1992): It is always best to assume the kernel is Gaussian, and it is better to rewrite formulae with the density placed inside of the operators. Although the first rule should be assumed as best practice, it would appear that while Gaussian kernels have good mathematical properties they do not have a compact support and require evaluations of exponential functions which is further discussed in Section 2.2. The second rule is used more frequently in modern $\mathrm{SPH}$, since it allows obtaining higher accuracy on the gradient of a quantity field (Kelager, 2006).

Calculating the pressure exerting on a particle is an important part of the SPH process, as it may influence how the particles react to one another, and therefore how the simulation behaves. To calculate the pressure at a particle the density must first be calculated, the pressure can then be determined using the ideal gas state equation $p=k \rho$, where $k$ is a gas stiffness constant that can be influenced by the number of particles in the fluid or the temperature. However, with the consideration of the rest density of the pressure, a modified equation had been rewritten to $p=k\left(\rho-\rho_{0}\right)$ (Müller et al., 2003). The revision to the equation is much more suited to the simulation of fluid as the initial equation was formalised to model gas, where particles emit a more repulsive reaction to neighbours. In fluid the particles would exhibit a more cohesive reaction and also have a constant massdensity at rest.

When the pressure at each particle is known, the 
application of the $\mathrm{SPH}$ rule to the pressure term $-\nabla p$ at particle $i$ is defined by

$$
\mathbf{P}_{i}=-\nabla p\left(\mathbf{r}_{i}\right)=-\sum_{j \neq i} m_{j} \frac{p_{j}}{\rho_{j}} \nabla W\left(\mathbf{r}_{i}-\mathbf{r}_{j}, h\right)
$$

However, this does not result in a symmetrical force, which is apparent when two particles interact. This is due to the particles only using one another to compute their respective forces. Changing the form to

$$
\mathbf{P}_{i}=-\sum_{j \neq i} m_{j} \frac{p_{i}+p_{j}}{2 \rho_{j}} \nabla W\left(\mathbf{r}_{i}-\mathbf{r}_{j}, h\right)
$$

to compute the pressure is not only fast and stable, but symmetry is guaranteed by using the arithmetic mean of the pressure between interacting particles (Müller et al., 2003).

The calculation of viscosity is another important factor to consider in SPH, each type of fluid has its own strength of viscosity, e.g. Water vs oil, and this must be modelled effectively. Viscosity is defined as the resistance to flow, and in SPH the viscosity coefficient $\mu$ defines the strength of how viscous the liquid is. In SPH terms the viscosity force term is defined by

$$
\mathbf{V}_{i}=\mu \nabla^{2} \mathbf{u}\left(\mathbf{u}_{i}\right)=\mu \sum_{j \neq i} m_{j} \frac{\mathbf{v}_{j}}{\rho_{j}} \nabla^{2} W\left(\mathbf{r}_{i}-\mathbf{r}_{j}, h\right)
$$

and much like the pressure force this is also an asymmetric force due to the velocity field variance between particles. A Similar form has also been introduced for force calculation, by including velocity differences as defined below (Müller et al., 2003).

$$
\mathbf{V}_{i}=\mu \sum_{j \neq i} m_{j} \frac{\mathbf{v}_{j}-\mathbf{v}_{i}}{\rho_{j}} \nabla^{2} W\left(\mathbf{r}_{i}-\mathbf{r}_{j}, h\right)
$$

This achieves symmetry because the viscosity forces only rely on the velocity differences, not the absolute velocities. Viscosity is further discussed in Section 3.4 .

Some of the equations necessary for implementing SPH have been detailed in this section, a pseudocode overview of a basic SPH simulation loop step provided by (Ihmsen et al., 2014) can be seen in Algorithm 1. This algorithm uses a state equation, so can be referred to as State-Equation SPH (SESPH).

\subsection{Kernel Functions and Smoothing Radius}

There are many similarities between the use of smoothing kernels in SPH, and difference schemes in finite difference methods (Monaghan, 1992). The selection of kernels for use within the SPH simulation is an important one since it may have a positive or negative effect on the accuracy produced, or the time taken

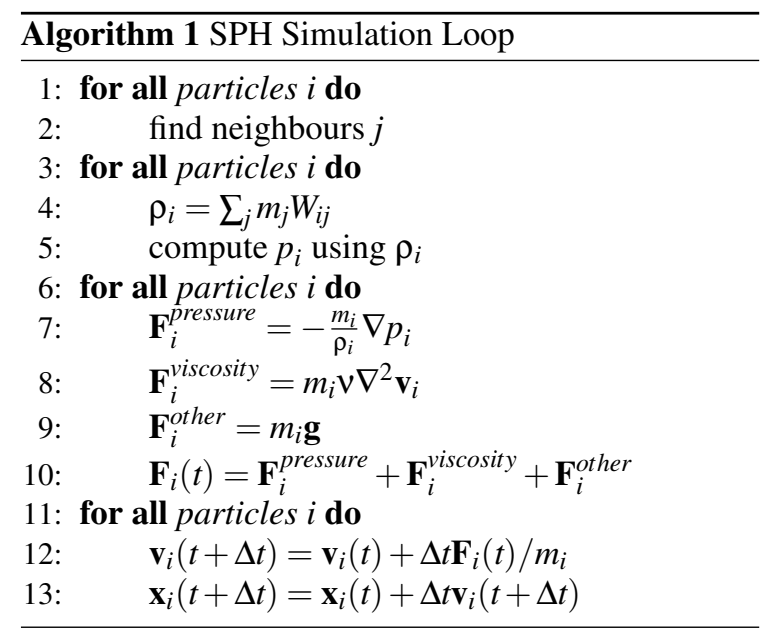

to perform the calculations and render the result. One advantage using kernels in SPH is the kernel can be calculated in a subroutine, meaning that interchanging kernels to assess their suitability to the simulation is trivial.

Monaghan suggested that a suitable kernel should be normalised

$$
W(\mathbf{r}, h)=W(-\mathbf{r}, h)
$$

and even

$$
\int_{\Omega} W(\mathbf{r}, h) d \mathbf{r}=1
$$

If both are satisfied then the interpolation is of second order accuracy. Furthermore, it is suggested that the kernel should have a limited or compact support range, this ensures that the kernel does not interact outside of the computational range of the defined radius. It is suggested that the kernel should also be positive to ensure that it is an averaging function, and if the kernel is even then rotational symmetry is enforced (Sporring et al., 2005). Another suggestion is that the kernel should also be monotonically decreasing, and that it should satisfy the Dirac delta function condition as $h \rightarrow 0$ (Liu et al., 2003), as defined below:

$$
\lim _{h \rightarrow 0} W\left(\mathbf{x}-\mathbf{x}^{\prime}, h\right)=\delta\left(\mathbf{x}-\mathbf{x}^{\prime}\right)
$$

The smoothing radius $h$, and smoothing kernel $\boldsymbol{W}$, used in SPH are important considerations to ensure a stable and robust simulation, and are subject to adjustment by changing the time or space. The problem of finding a suitable smoothing radius is analogous to deciding on the amount of particles that a SPH simulation should include. If $h \rightarrow \infty$ then the simulation can become unstable due to the kernel weighting particle contributions less at the centre of the search radius. Conversely, if $h \rightarrow 0$ then not enough particles will be used in the weighting performed by the 
kernel, and the results again will be imprecise. Although some methods of finding a suitable value of $h$ exist, some experimentation may be required to find the optimum level at which the simulation behaves accordingly, while maintaining low computation times. Considering $h$ as a spherical radius, calculate the optimum size such that $x$ particles comfortably fill the spherical volume, then choose a suitably small number of $x$ such that the fluid simulation is stable and behaves accordingly, while still maintaining the properties of the chosen fluid (Kelager, 2006).

\subsection{Boundary Conditions}

Fluid simulation implementing SPH should effectively detect and respond to collisions between the fluid particles, known as fluid-fluid interaction, and with the boundary (container) and any rigid objects or meshes placed in the scene, known as fluid-rigid interaction. A simple method of collision response to fluid-rigid interaction is to reflect the colliding particle and its current velocity perpendicularly to the object surface, generally calculated via the surface normal of the object at the point of contact. The simplicity of this method is also its disadvantage, when the boundary comprises of a simple flat plane this method can be reliable for small simulations. But when more complex shapes are used or the simulation size is large it can lead to discrepancies in calculations, where the particles behave erratically or simply pass through the object.

Furthermore, Having a perfectly elastic collision is generally not advised, since fluid does not behave this way in nature, and as such the velocity should be subject to division or multiplication by a restitution coefficient. There are a variety of methods available to effectively manage particle interaction with the boundary and other rigid objects in the simulation space, since computing interaction via kernels can cause some instability and clip parts of the object entirely. Many of these methods generate static particles, known as ghost or mirror particles, at the surface of the object that have a repulsion force to repel any incoming fluid particles. In the next paragraph some of the more recent contributions to the study of boundary handling in SPH will be discussed.

One such method involves the addition of an interface between the fluid and the boundary, defined as a surface between the two adjacent materials, and then define three boundary conditions (Müller et al., 2004):

1. No-Penetration Condition: No fluid is allowed to cross the boundary,

$$
\left(\frac{\partial}{\partial t} \mathbf{u}-\mathbf{v}\right) \cdot \mathbf{n}=0 \text { at the boundary } \Gamma
$$

2. No-Slip Condition: Models friction between the fluid and the solid,

$$
\left(\frac{\partial}{\partial t} \mathbf{u}-\mathbf{v}\right) \times \mathbf{n}=0 \text { at the boundary } \Gamma
$$

3. Actio $=$ Reactio: The traction forces of the solid must be opposite to that of the fluid on the boundary $\Gamma$,

$$
g_{s}=\sigma_{s} \mathbf{n}=\sigma_{f}(-\mathbf{n})=-g_{f}
$$

By incorporating density estimated at the boundary into the pressure forces acting on the fluid, while maintaining the ability to predict and correct the particle positions. It is also possible to prevent particle stacking and smoother density distributions at the boundaries (Ihmsen et al., 2010). By replacing the boundary entirely with particles which interact with the fluid with a prescribed force, simulations can easily replicate interaction between the fluid mass and any rigid bodies, that may also float (Monaghan, 2005). By sampling the solid boundary simulating air particles with "ghost particles", this can correctly simulate cohesion between the fluid and solid objects while reducing the amount of artifacts produced (Schechter and Bridson, 2010). A similar approach uses "mirror-particles", the domain is discretised using a set of triangles for ease of calculating the normal directions, the mirror-particles are generated when a particle is found to be within a certain threshold of the triangle (Napoli et al., 2015).

Similarly, a coupled, dynamic solid boundary treatment approach can be used where the boundaries of the domain are padded with an inner layer of particles that exhibit a repulsive force, and two outer layers of ghost particles (Chen et al., 2015). Some of these approaches can suffer with inhomogeneous particle sampling, but this can be solved by deriving new equations which consider the boundary particles relative contribution to a physical quantity. This can also improve the particle initialisation at complex boundaries and the boundary resampling of any dynamic objects in the simulation (Akinci et al., 2012).

\subsection{Nearest Neighbour Searching}

In order to effectively calculate forces between particles SPH necessitates using a Nearest Neighbour search algorithm to locate any particles that may influence the root particle. Illustrated in Figure 2, Nearest Neighbour searching is an extremely computationally expensive operation, and as such can have a largely 


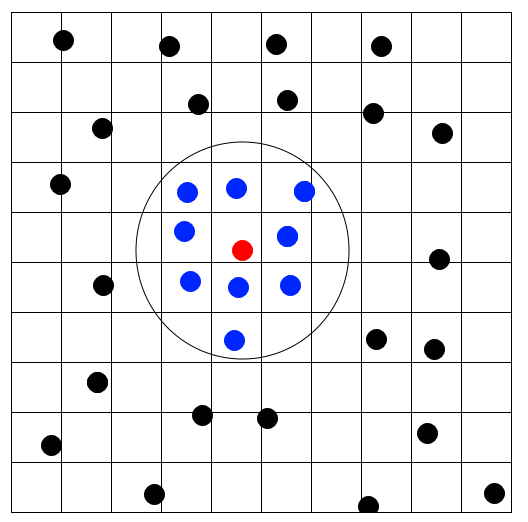

Figure 2: 2D grid-based nearest neighbours: the root particle shown in red and its neighbouring particles shown in blue within a defined search radius, the black particles outside would not be considered in pressure and viscosity calculations.

negative impact on the time taken to process each frame. It is advised to utilise a method of spatial decomposition so that the entire set of particles need not be iterated over to find a specific instance. Spatial decomposition offers a method of dividing the working space of the simulation into smaller sections, generally referred to as voxels or cells, that can be accessed directly using a divisor such as position in 3D space, or lookup key.

A popular method of Nearest Neighbour searching uses a 3D uniform grid, a bounding cube contains the particles, and a twice division strategy to calculate the optimum edge length of the grid cells. The expense of performing sorting operations on the set can be reduced by using only certain query points, which in turn reduces the necessary number of distance calculations required (Zhao et al., 2014) . Another popular method is spatial hashing, the effectiveness is bound by the speed with which the unique hash keys used to represent each grid cell can be generated. But care must be taken to ensure that there are no "hash collisions", where multiple hash keys map to the same grid cell (Kelager, 2006). By using a sliced data structure and the GPU to construct and access the stored values, the computational space is sliced into a number of two-dimensional planes and an index, and a dynamic grid is constructed to fit the particle distribution with some margin (Harada et al., 2007).

Some methods can be executed entirely on the GPU, using table lookup based Z-Indexing for range queries and a virtual indexing grid for spatial subdivision. The Z-Index is used to sort the particles and any found within a power-of-two sized aligned block are considered neighbours (Goswami et al., 2010). In comparison with a number of techniques implementing uniform grids that include index, parallel and Z-
Index sorting, and spatial and compact hashing. It shows that Z-Indexing and compact hashing are both equally efficient methods, where the memory consumption of the preceding scales with the domain, and that of the proceeding with the number of particles (Ihmsen et al., 2011) .

\subsection{Numerical Time Integration \& Time Step}

There are many different methods of numerical integration, and for clarity the most regularly implemented will be briefly discussed.

The Implicit Euler scheme is, contrary to its name, a semi-implicit numerical integration method and is based on the more commonly used explicit Euler scheme. Unlike the explicit Euler, where the position and velocity are updated in parallel, the positional update utilises the result of the velocity calculation

$$
\mathbf{u}_{t+\Delta t}=\mathbf{u}_{t}+\Delta t \mathbf{a}_{t}
$$

to estimate the new particle position

$$
\mathbf{r}_{t+\Delta t}=\mathbf{r}_{t}+\Delta t \mathbf{u}_{t}
$$

The Leap-Frog scheme utilises the structure of the implicit Euler method, and its name is derived from the manner in which the velocities and positions "leap" over one another. The initial velocity offset is calculated using a Euler step

$$
\mathbf{u}_{-\frac{1}{2} \Delta t}=\mathbf{u}_{0}-\frac{1}{2} \Delta t \mathbf{a}_{0}
$$

with the regular velocity calculation given by

$$
\mathbf{u}_{t+\frac{1}{2} \Delta t}=\mathbf{u}_{t-\frac{1}{2} \Delta t}+\Delta t \mathbf{a}_{t}
$$

and the position calculated by

$$
\mathbf{r}_{t+\Delta t}=\mathbf{r}_{t}+\Delta t \mathbf{u}_{t+\frac{1}{2} \Delta t}
$$

In order to estimate a given velocity at arbitrary time $t$ a midpoint approximation can be used

$$
\mathbf{u}_{t} \approx \frac{\mathbf{u}_{t-\frac{1}{2} \Delta t}+\mathbf{u}_{t+\frac{1}{2} \Delta t}}{2}
$$

Deciding on the most appropriate time step is an important factor when considering time integrating methods, since it can effect the stability of the integration. Due to the manner in which SPH utilises regular differential equations, any stable method of time stepping can be used. However, when there is an absence of dissipation it would be beneficial to use a symplectic integrator such as the Verlet second order integrator (Monaghan, 2005). Comparing Weakly-Compressible SPH with Incompressible 
$\mathrm{SPH}$, the choice of time step is separate for each but chosen by the same expression, to satisfy the minimum of three conditions: the Courant-FriedrichsLevy condition, and the mass and viscosity force conditions (Lee et al., 2008). However, it is also possible to control the time-step by using the aforementioned CFL condition, gravity, viscosity and drag terms (Monaghan, 2005).

\subsection{Compressibility and SPH}

The original formulation of the SPH method was designed to model compressible flow problems, but implementing incompressibility is an important factor when creating realistic simulations. Enforcing incompressibility can be a computationally expensive step, and can be achieved using a variety of different methods. One of the simpler methods solves an equation of state using the density to derive the pressure. This however has its disadvantages, one being that the time step has to be extremely small, and another that nonphysical pressure fluctuations caused by erroneous density calculations can lead to numerical instability in the simulation. Fully incompressible SPH aims to rectify these issues by treating pressure and viscosity forces separately. The pressure is calculated by projecting the intermediate velocity field, found by integrating the field forward in time without enforcing incompressibility, onto a divergence-free space by solving a derived pressure Poisson equation (Cummins and Rudman, 1999). Other methods include WeaklyCompressible SPH (WCSPH), Implicit Incompressible SPH (IISPH), Predictive-Corrective Incompressible SPH (PCISPH), and Local-Poisson SPH (LC$\mathrm{SPH})$.

By experimenting with a variety of simulations including 2D lid-driven cavity flow, flow around a bluff, and a series of dam-breaks, Incompressible SPH out-performed the Weakly-Compressible, yielding more reliable results with smoother velocity and pressure fields (Lee et al., 2008). Further methods have been applied to enhance the implementation of Incompressible SPH, such as Moving Particle SemiImplicit methods and error compensating source of the pressure Poisson equation (Gotoh et al., 2014).

Recently, a method named Predictive-Corrective Incompressible SPH combining the advantages of Weakly-Compressible SPH and Incompressible SPH, low computational cost per update and a larger time step has been presented (Solenthaler and Pajarola, 2009). It uses a prediction-correction scheme for propagating estimated density values through the fluid until a user defined density variation limit is reached, and updates the particle pressures such that incom- pressibility is enforced. Compared with WCSPH, PCISPH achieves similar results with less computation, and also allows the use of much larger time steps without sacrificing the stability of the simulation. Recent studies have shown that approaches combining Implicit-Incompressible SPH with another popular method of fluid simulation known as Fluid-ImplicitParticle (FLIP), have several advantages compared with regular SPH including low memory usage, computation time and larger time steps. The combination of the two methods counteract the disadvantages of either individually, with low memory consumption for large scale simulations, and low computational complexity (Cornelis et al., 2014).

To avoid the computational cost of solving a Poisson pressure equation globally, a Local Poisson SPH method that retains the large time step of Incompressible SPH has been introduced. It effectively eliminates the large density deviations arising from solid boundary treatment (He et al., 2012)

\section{Experimental Applications}

Recent research on SPH is primarily concerned with refining, or detailing new advances which improve the algorithms used in creating the various simulations. Although some research focuses on specific scenarios where SPH is used such as viscous fluids, multiphase fluids, erosion modelling, and interactive fluids. Some of the recent research and developments will be discussed in the proceeding subsections.

\subsection{Multiphase Flows}

Many SPH simulations are based upon "single-phase" flows, which is a single liquid interacting with boundaries or obstacles placed in the scene. Fluids with more than one phase are called multiphase, and can be classified as miscible, where the phases can freely mix with one another; or immiscible where the phases are separate, and cannot initiate a chemical reaction between them. An example of miscible flow would be the mixing of two liquids with different viscosities e.g. combining oil and vinegar, and immiscible flow e.g. lava flows, water at boiling point. The separating axis in multiphase flows is often referred to as an interface of zero thickness that balances the separation of two phases using surface tension (Chen et al., 2015).

After simulation of lava flows and the dusty gas produced in volcanic eruptions, it was noted that $\mathrm{SPH}$ is particularly suitable for modelling multiphase flow due to the ease of modification to handle gas, solid or 
liquid phases, achieved with the inclusion of an energy equation (Monaghan and Kocharyan, 1995). By further modifying the particle approximation step, introducing a density re-initialisation treatment to correct any mass discontinuities occurring at the interfaces can also improve multi-phase flow SPH. Particles residing close to the interfaces between phases are treated as ghost particles for any particles from neighbouring phases (Chen et al., 2015). In their research, refinement of the mixture model also improves multi-phase simulation where the reliance on tracking interfaces between the phases is replaced by representing the phases with their volume fractions (Ren et al., 2014). Each phase has its own set of particles that carry the mixture mass, velocity, and any physical qualities of the phase to discretise the multiphase system.

\subsection{Bubbles, Foam and Splashing}

In fluid simulation the representation of the fluid is of foremost importance. The addition of features that fluid creates in its movement such as bubbles, waves, foam, water splashing and turbulence is also important in creating realistic visualisations. Some may be created when the fluid interacts with solid objects in its environment, or when something is introduced to the fluid e.g. a whisk or large object falling into the fluid. The computation required to represent these features can be quite complex depending on the simulation, but the visualisation thereof is also a complex task and can require a variety of different techniques to render accurately.

In general, bubbles are created when air particles get trapped within the fluid, in the case of water which has a low viscosity these bubbles would quickly rise to the surface of the liquid possibly becoming foam. In a more viscous liquid the bubbles may stay in place creating an air pocket. This is due to a difference in density, or rest-density, between the particles. When two particle bodies of different density are mixed the difference will cause the less dense fluid to rise. Bubbles are able to be simulated using SPH by generating air particles where air pockets are likely to form in the fluid, this can be extended to simulate boiling water through phase transitions by tracking the temperature and changing the type and density of the particles dynamically (Müller et al., 2005). Multi-phase SPH formulations are well suited to simulating bubbles and foam, using a saturated function for volume allows smaller and larger bubbles to behave differently, and the inclusion of a drag force can effectively simulate the two-way interaction between the phases. When air particles reach the surface of the fluid body they are treated as foam particles that have a finite lifespan before being removed. Detecting particles that have breached the surface can be achieved using a smoothed colour field, or comparing the number of neighbours using a defined threshold (Akinci et al., 2011).

Splashing or sloshing occurs when fluid particles leave the main body of fluid, this can be the result of fluid interaction with solid boundaries or objects, and user interaction with the fluid. SPH is able to simulate some instances of splashing in its original form, however this can be improved to simulate a much more realistic representation, such as the introduction of an error compensating source of the Poisson pressure equation, and a higher order laplacian (Gotoh et al., 2014). Apart from the robustness and stability that SPH can offer for most of the simulations, to simulate sparsely sampled thin features in free surface flows e.g. splashing, SPH might encounter difficulty towards failure. By applying the combination of a free surface energy function based surface tension force schemes, efficient air pressure calculation, and geometry aware anisotropic kernels used to filter internal pressure estimated at two scales, the simulation has been enhanced and improved (He et al., 2014).

\subsection{Interactive Fluids}

Fluid interaction can take place between fluid particles and a wide number of different mediums including deformable solids or bodies, free surfaces, and particles representing different fluids or gases. SPH is able to be combined with a Finite Element approach to model the interactivity of fluid particles and deformable solids, where boundary particles are placed at the object surface according to Gaussian quadrature rules (Müller et al., 2004).

Particle-air interaction with SPH can be effectively modelled by the calculation of the surfacetension and adhesion forces, which removes the necessity of surface tracking and the use of ghost or virtual particles (Akinci et al., 2013b). Another method applies the generation of air particles where air pockets are likely to be formed in the liquid. The inclusion of temperature calculations to adjust particle types and densities means phenomena such as boiling water can also be modelled (Müller et al., 2005).

Fluid interaction with soluble objects where the object is composed entirely of particles, can be modelled with SPH via the inclusion of a dissolution model. During dissolution the object particle concentration is transferred to the adjacent fluid particles until the object particle is completely dissolved, at which point it detaches from the object (Kim and 
Park, 2014).

\subsection{Viscous, Elastic Fluids and Objects}

Viscous, and Viscoelastic fluids in SPH include substances such as oil, honey, blood, and lava. Fluids can be subject to dynamic alteration due to temperature changes including melting, freezing into a solid, and viscosity fluctuations in flow which will change the behaviour and appearance of the fluid. Although SPH can model viscous fluids, a problem known as tensile instability can arise due to the cohesive pressure which can cause the particles to cluster or become sparsely distributed. The tensile instability problem is able to be rectified with the use of a hyperbolic-shaped kernel that possesses non-negative second derivatives, which ensures even distribution of particles in the fluid (Yang et al., 2014).

The simulation of bubbles and air particles in particularly viscous liquids can be problematic, but through the use of multi-phase SPH formulations it is possible to compute the two-phase flow inside and outside of the bubble which addresses the large density differences, and surface tension is able to be enforced using a Continuum Surface Force. Improving the efficiency and robustness of viscous fluid simulations using SPH has been achieved via the implicit integration of viscosity, and the conversion to a sparse linear system with a symmetric positive matrix (Takahashi et al., 2015). Other improvements include the reconstruction of the velocity field from the target velocity gradient, where any density corrections from the preceding pressure projection stage are preserved meaning only one pressure projection step is required (Peer et al., 2015). Both revisions improve the simulation reliability, and results, with the phenomena of buckling and coiling and multi-phase viscous liquid mixing correctly represented.

Elastic-solid coupling using SPH fluids can be achieved by sampling the triangulated surfaces of solids using boundary particles, but can suffer from problems when using deformable boundaries such as spatial and temporal discontinuities and particle leaking through boundaries. A method of countering this is through the use of specific surface tension and adhesion forces which do not rely on the introduction of ghost particles (Akinci et al., 2013b).

Using SPH to model objects or solids in simulations is also popular, finding use in deformable objects and meshes, and elastic and plastic objects. As the object can be represented entirely by the particles, processes such as handling large deformations, adding special conditions such as repulsion forces, or simulating the fluid body interacting with the solid is relatively simple. A method often used in Finite Element Methods, the corotational formula, has been adapted for use in modelling meshless deformable solids with SPH. The rotations in the deformation field are computed using a variant of the shape matching approach adapted for use with SPH (Becker et al., 2009). Compared with similar methods, the corotational approach not only improves the realistic behaviour of the simulation, but also the range of elastomechanical properties that can be simulated.

\section{Current Trends in SPH}

Since its creation, SPH has been adapted and modified to suit a variety of research areas. With extensions to the original model it has proved extremely adaptive, finding use in various areas including sound and acoustics research, naval engineering and bioengineering. This section aims to look at a selected number of areas currently popular with researchers in fluid dynamics.

\subsection{Surface Reconstruction}

Surface reconstruction is an important research topic in SPH. The body of the fluid is represented by the particles in the simulation, but to render these as a body of fluid the particle surfaces must be extracted. This is a computationally expensive operation when constructing a high resolution, detailed and artifact free surface from large sets of particles and is often seen as a bottleneck due to the complexity and high memory usage.

Some of the most widely adopted methods involve algorithms such as marching cubes or tiles, and raytracing. The main problem with marching cubes is the high memory usage and wastage, which can impact execution times and results. The Histogram Pyramid Marching Cubes algorithm removes any cells which contain no triangles which improves on the memory consumption, and can also be implemented on the GPU, greatly improving execution times (Huang et al., 2015).

There are also methods that reconstruct the surfaces from grids and anisotropic kernels. With each particle represented by anisotropic kernels, marching cubes is used to construct a mesh that approximates the fluid surface, with additional diffusion smoothing steps to account for any bumpiness on the surface (Yu and Turk, 2010).

Three-level grids have been used for surface reconstruction on the CPU (Akinci et al., 2013a) and more recently on the GPU, where the execution time 
was vastly improved due to parallelisation. Using a grid can lead to cracks in the approximated surface which appear in the common faces between adjacent grid cells, so techniques must account for this with some approaches dealing with it procedurally, detecting and filling cracks at runtime (Du and Kanai, 2014). Surface reconstruction using Volume rendering makes further use of grids, resampling the particle quantities onto a view volume sized $3 \mathrm{D}$ grid which is stored as a 3D texture which can be stored and used on the GPU for ray casting (Fraedrich et al., 2010).

\subsection{GPU Utilisation}

The computational complexity of fluid simulation is a challenging area and one that prevents truly real-time simulations from achieving good graphical fidelity. To correctly model fluid the simulation should comprise of a relatively large number of particles and the complexity scales with this. SPH is less dependent on data and well suited for parallelisation, as are methods it relies on such as Nearest Neighbour searching and Surface Extraction/Reconstruction. Parallel processing can be achieved using multi-core CPUs and multithreading, field programmable gate arrays (FPGA), Gravity Pipes (GRAPE) and the GPU. Multicore CPUs and powerful GPUs are now commonplace in most personal computers available and as such are the most accessible forms of parallel computing, with the GPU exhibiting the best price-performance ratio. The parallelisation of SPH has seen an increasing amount of research as the programmability of the GPU has become more accessible using languages such as NVIDIA's CUDA and OpenCL.

Approaches executing the entire simulation on the GPU can be achieved by removing the data dependencies and changing the computational model from "gathering" to "distributing". Adaptive sampling is used to allow focusing of computation on specific areas in the fluid, and particle merging to reduce the overall number of particles (Zhang et al., 2008). GPU implementations can also benefit from optimisations regarding how positional and neighbourhood data is stored on the GPU (Rustico et al., 2014), and ensuring data representation is optimised for use by the GPU. For instance, particle data can maintain contiguity if an Array of Structures (AoS) format is used. But as not all particle attributes are accessed simultaneously a Structure of Arrays (SoA) format achieves a higher memory bandwidth on the GPU (Nie et al., 2015).

To extend the previous work executed on single GPU systems (Hérault et al., 2010), an optimisation method was utilised in considering updating how positional and neighbourhood data is stored to better suit
GPU memory access, and the replacement of deprecated functions no longer supported in CUDA (Rustico et al., 2014). The proposed revision with a single GPU implementation is more than twice as fast as the original.

\section{Conclusions}

Smoothed Particle Hydrodynamics is a fully meshfree particle based method, where the particles carry the material properties of the medium it is simulating (fluid, gases). This paper has presented a survey of the current state of SPH research and techniques, some of the history of the SPH method, its application in various fields, and some of the more recent improvements.

The largest issue still facing researchers of SPH is the computational overhead. Many of the simulations presented in papers involving a very large number of particles or complex procedures are prerendered. Advances made in computer hardware are having a positive effect on this issue, and are enabling researchers to devise new strategies and techniques to improve the execution times, but it will likely not completely resolve this issue. Furthermore, refinement of the algorithms used and development of new algorithms will likely assist in the contribution of reducing the complexity. Simulations executed on the GPU is an area that shows a lot of promise, as SPH is well suited to parallel execution this offers a large speed advantage over traditional execution on the CPU.

As SPH is a purely particle based method, to simulate actual fluid the particles need to be rendered as such. Surface reconstruction is a technique for achieving visualisation of fluids and its complexity scales with the number of particles used in the simulation. Furthermore, other time consuming calculations such as lighting and shadow effects, or surface reflections used to create an aesthetically pleasing liquid are an important consideration. Real-time simulations executed on the GPU using around 30,000 particles can maintain a steady average frame rate of around 50 frames per second (fps), adding surface extraction and rendering can reduce this by between 60 $80 \%$. So, efficient rendering could be considered a bottleneck in fluid simulation using SPH and would benefit from further research. 


\section{REFERENCES}

Akinci, G., Akinci, N., Oswald, E., and Teschner, M. (2013a). Adaptive surface reconstruction for sph using 3-level uniform grids.

Akinci, M., Julian, I., Gizem, B., and Teschner, M. (2011). Animation of air bubbles with sph.

Akinci, N., Akinci, G., and Teschner, M. (2013b). Versatile surface tension and adhesion for sph fluids. ACM Transactions on Graphics, 32.6:182.

Akinci, N., Ihmsen, M., Akinci, G., Solenthaler, B., and Teschner, M. (2012). Versatile rigid-fluid coupling for incompressible sph. ACM Transactions on Graphics (TOG), 31(4):62.

Altomare, C., Crespo, A. J., Domnguez, J. M., GmezGesteira, M., Suzuki, T., and Verwaest, T. (2015). Applicability of smoothed particle hydrodynamics for estimation of sea wave impact on coastal structures. Coastal Engineering, 96:1-12.

Becker, M., Ihmsen, M., and Teschner, M. (2009). Corotated sph for deformable solids. $N P H$, pages 27-34.

Chen, Z., Zong, Z., Liu, M. B., Zou, L., Li, H. T., and Shu, C. (2015). An sph model for multiphase flows with complex interfaces and large density differences. Journal of Computational Physics, pages 169-188.

Cornelis, J., Ihmsen, M., Peer, A., and Teschner, M. (2014). Iisph-flip for incompressible fluids. Computer Graphics Forum, 33(2).

Cummins, S. J. and Rudman, M. (1999). An sph projection method.

Du, S. and Kanai, T. (2014). Gpu-based adaptive surface reconstruction for real-time sph fluids.

Fraedrich, R., Auer, S., and Westermann, R. (2010). Efficient high-quality volume rendering of sph data. Visualization and Computer Graphics, 16.6:1533-1540.

Gingold, R. A. and Monaghan, J. J. (1977). Smoothed particle hydrodynamics: theory and application to nonspherical stars. Monthly notices of the royal astronomical society, 181.3s:375-389.

Goswami, P., Schlegel, P., Solenthaler, B., and Pajarola, R. (2010). nteractive sph simulation and rendering on the gpu. Proceedings of the 2010 ACM SIGGRAPH/Eurographics Symposium on Computer Animation.

Gotoh, H., Khayyer, A., Ikari, H., Arikawa, T., and Shimosako, K. (2014). On enhancement of incompressible sph method for simulation of violent sloshing flows. Applied Ocean Research, 46:104-115.

Harada, T., Koshizuka, S., and Kawaguchi, Y. (2007). Sliced data structure for particle-based simulations on gpus. Proceedings of the 5th international conference on Computer graphics and interactive techniques in Australia and Southeast Asia.

He, X., Liu, N., Li, S., Wang, H., and Wang, G. (2012). Local poisson sph for viscous incompressible fluids. Computer Graphics Forum, 31(6):1948-1958.
He, X., Wang, H., Zhang, F., Wang, H., Wang, G., and Zhou, K. (2014). Robust simulation of sparsely sampled thin features in sph-based free surface flows. ACM Transactions on Graphics (TOG), 34.

Hérault, A., Bilotta, G., and Dalrymple, R. A. (2010). Sph on gpu with cuda. Journal of Hydraulic Research, 48.S1:74-79.

Huang, C., Zhu, J., Sun, H., and Wu, E. (2015). Paralleloptimizing sph fluid simulation for realistic vr environments. Computer Animation and Virtual Worlds, 26(1):43-54.

Ihmsen, M., Akinci, N., Becker, M., and Teschner, M. (2011). A parallel sph implementation on multi-core cpus. Computer Graphics Forum, 30:99-112.

Ihmsen, M., Akinci, N., Gissler, M., and Teschner, M. (2010). Boundary handling and adaptive timestepping for pcisph. Workshop on virtual reality interaction and physical simulation VRIPHYS.

Ihmsen, M., Orthmann, J., Solenthaler, B., Kolb, A., and Teschner, M. (2014). Sph fluids in computer graphics. Eurographics - State of the Art Reports.

Kelager, M. (2006). Lagrangian fluid dynamics using smoothed particle hydrodynamics. University of Copenhagen. Denmark.

Kim, S. and Park, J. (2014). A sph-based dissolution behavior model for real-time fluid-solid interaction. $S I G$ GRAPH Asia 2014 Posters.

Lee, E.-S., Moulinec, C., Xu, R., Violeau, D., Laurence, D., and Stansby, P. (2008). Comparisons of weakly compressible and truly incompressible algorithms for the sph mesh free particle method. Journal of computational physics, 227:8417-8436.

Liu, M. B., Liu, G. R., and Lam, K. Y. (2003). Constructing smoothing functions in smoothed particle hydrodynamics with applications. Journal of Computational and applied Mathematics, 155.2:263-284.

Lucy, L. B. (1977). A numerical approach to the testing of the fission hypothesis. Astronomical Journal, 82:1013-1024

Monaghan, J, J. and Kocharyan, A. (1995). Sph simulation of multi-phase flow. Computer Physics Communications, 87:225-235.

Monaghan, J. J. (1992). Smoothed particle hydrodynamics. Annual review of astronomy and astrophysics, 30:543-574.

Monaghan, J. J. (2005). Smoothed particle hydrodynamics. Reports on progress in physics, 68.8:1703-1759.

Müller, M., Charypar, D., and Gross, M. (2003). Particle-based fluid simulation for interactive applications. Proceedings of the 2003 ACM SIGGRAPH/Eurographics symposium on Computer animation.

Müller, M., Schirm, S., Teschner, M., Heielberger, B., and Gross, M. (2004). Interaction of fluids with deformable solids. Computer Animation and Virtual Worlds 15, no 3-4:159-171. 
Müller, M., Solenthaler, B., Keiser, R., and Gross, M. (2005). Particle-based fluid-fluid interaction. Proceedings of the 2005 ACM SIGGRAPH/Eurographics symposium on Computer animation, pages 237-244.

Napoli, E., Marchis, M. D., and Vitanza, E. (2015). Panormus-sph. a new smoothed particle hydrodynamics solver for incompressible flows. Computers and Fluids, 106:185-195.

Nie, X., Chen, L., and Xiang, T. (2015). Real-time incompressible fluid simulation on the gpu. International Journal of Computer Games Technology.

Pan, W., Daily, M., and Baker, N. A. (2015). Numerical calculation of protein-ligand binding rates through solution of the smoluchowski equation using smoothed particle hydrodynamics. BMC biophysics, 8.1.

Peer, A., Ihmsen, M., Cornelis, J., and Teschner, M. (2015). An implicit viscosity formulation for sph fluids. $A C M$ Transactions on Graphics, 34(4):114.

Ren, B., Li, C., Yan, X., Lin, M. C., Bonet, J., and Hu, S. M. (2014). Multiple-fluid sph simulation using a mixture model. ACM Transactions on Graphics, 33 no. 5:171.

Rustico, E., Bilotta, G., Herault, A., Del Negro, C., and Gallo, G. (2014). Advances in multi-gpu smoothed particle hydrodynamics simulations. Parallel and Distributed Systems, IEEE Transactions, 25(1):43-52.

Schechter, H. and Bridson, R. (2010). Ghost sph for animating water. ACM Transactions on Graphics (TOG), 31(4):61.

Solenthaler, B. and Pajarola, R. (2009). Predictivecorrective incompressible sph. ACM Transactions on Graphics (TOG), 28(3).

Sporring, J., Henriksen, K., and Dohlmann, H. (2005). Physics-based animation. Hingham: Charles River Media.

Takahashi, T., Dobashi, Y., Fujishiro, I., and Nishita, T., a. L. M. (2015). Implicit formulation for sphbased viscous fluids. Computer Graphics Forum, 34(2):493502.

Yang, X., Liu, M., and Peng, S. (2014). Smoothed particle hydrodynamics modeling of viscous liquid drop without tensile instability. Computers and Fluids, 92:199208.

Yu, J. and Turk, G. (2010). Reconstructing surfaces of particle-based fluids using anisotropic kernels. ACM Transactions on Graphics, 32.1:5.

Zhang, Y., Solenthaler, B., and Pajarola, R. (2008). Adaptive sampling and rendering of fluids on the gpu. Proceedings of the Fifth Eurographics/IEEE VGTC conference on Point-Based Graphicsg.

Zhang, Y., Zhang, T., Li, T., and Wang, P. (2015). Smoothed particle hydrodynamics approach for modeling sound of a rigid body falling on water. The Journal of the Acoustical Society of America, 137(4):2403.

Zhao, J., Long, C., Xiong, S., Liu, C., and Yuan, Z. (2014). A new $\mathrm{k}$ nearest neighbours algorithm using cell grids for $3 \mathrm{~d}$ scattered point cloud. Elektronika ir Elektrotechnika, 20(1):81-87. 